\title{
USO DE SCANNER EM ESPECTROFOTOMETRIA DE ABSORÇÃO MOLECULAR: APLICAÇÃO EM EXPERIMENTO DIDÁTICO ENFOCANDO A DETERMINAÇÃO DE ÁCIDO ASCÓRBICO
}

\author{
Marcos S. Gomes, Lilian C. Trevizan e Joaquim A. Nóbrega* \\ Departamento de Química, Universidade Federal de São Carlos, CP 676, 13560-970 São Carlos - SP, Brasil \\ Marcos Y. Kamogawa \\ Departamento de Química, Escola Superior de Agricultura "Luiz de Queiroz", Universidade de São Paulo, \\ Piracicaba - SP, Brasil
}

Recebido em 27/3/07; aceito em 14/11/07; publicado na web em 13/8/08

\begin{abstract}
MOLECULAR ABSORPTION SPECTROPHOTOMETRY USING A SCANNER: PROPOSAL OF A DIDACTIC EXPERIMENT FOR ASCORBIC ACID DETERMINATION. Quantitative analysis of colored substances is generally performed using a spectrophotometer for detection of radiation. However, the combination of digital images and mathematical conversion of colors is an alternative for quantitative procedures based on colored chemical reactions. This work proposes a didactic experiment using a scanner for determination of ascorbic acid in medicines based on the formation of the Prussian blue complex. Results were in good agreement with a conventional spectrophotometer method and the procedure is attractive for instrumental analysis undergraduate courses.
\end{abstract}

Keywords: spectrophotometry; scanner; ascorbic acid.

\section{INTRODUÇÃO}

Análises químicas são realizadas cotidianamente para determinação de uma grande variedade de substâncias. Análises clínicas, nutricionais e controle de qualidade são apenas algumas das aplicações da química analítica no nosso dia-a-dia. Em algumas situações, como por exemplo, nas análises preliminares de doping realizadas em atletas, o analista deseja apenas detectar a presença de uma determinada substância. Nesses casos, a determinação é denominada qualitativa. Por outro lado, quando é necessário estabelecer a quantidade exata da substância a ser analisada executa-se uma análise quantitativa.

Cursos de graduação em química apresentam em suas grades curriculares disciplinas específicas de química analítica e muitos deles dispõem de laboratórios para realização de experimentos voltados para análises quantitativas de produtos de uso rotineiro. Alguns dos experimentos comumente propostos baseiam-se na capacidade que algumas substâncias apresentam para formar compostos coloridos. O ácido ascórbico (vitamina C), por exemplo, reage com hexacianoferrato de potássio $\left(\mathrm{K}_{3}\left[\mathrm{Fe}(\mathrm{CN})_{6}\right]\right)$ na presença de $\mathrm{Fe}(\mathrm{III})$ para formar um composto colorido, denominado azul da Prússia. ${ }^{1} \mathrm{~A}$ intensidade da coloração azul da solução depende da concentração de ácido ascórbico presente na amostra. Dessa forma, a medida da intensidade da coloração possibilita a análise quantitativa desse composto em uma determinada amostra. Esse procedimento foi adaptado por Nóbrega e Lopes em um sistema de injeção em fluxo com detecção espectrofotométrica. ${ }^{2}$

A maneira mais comum de determinar a quantidade da substância colorida é através do uso de espectrofotômetros que operam nas regiões do ultravioleta e visível do espectro eletromagnético. Esses equipamentos apresentam, basicamente, uma fonte contínua (por exemplo, uma lâmpada de tungstênio) que emite radiação, um monocromador para seleção de uma faixa estreita de comprimentos de onda que atingem a solução contendo o analito, um compartimento para posicionamento da amostra e um dispositivo para detecção da medida da intensidade de radiação. ${ }^{3,4}$ Equipamentos mais modernos

\footnotetext{
*e-mail: djan@ufscar.br
}

podem armazenar e manipular os dados obtidos com auxílio de microcomputadores.

Moléculas e íons presentes são capazes de absorver fótons e são então promovidos do estado fundamental para um estado excitado mais energético. A quantidade de energia absorvida é proporcional à concentração da espécie na solução, possibilitando a quantificação. Essa relação é conhecida como Lei de Lambert-Beer e é baseada na medida da transmitância $T=\mathrm{P} / \mathrm{P}_{0}\left(\mathrm{P}_{0}\right.$ é a potência do feixe de radiação incidente e $\mathrm{P}$ é a potência do feixe de radiação após atravessar a solução contendo a espécie de interesse) ou absorbância $A$ de espécies absorventes, átomos, moléculas ou íons, contidas em uma região de observação com um caminho óptico de $b \mathrm{~cm}$. A concentração $c$ de um analito absorvente está relacionada linearmente à absorbância, onde $e$ representa a absortividade molar, que é uma grandeza característica da espécie absorvente, cuja magnitude depende do comprimento de onda e da monocromaticidade da radiação incidente, da secção de choque da espécie absorvente e da probabilidade de transição eletrônica. A lei de Lambert-Beer pode ser representada por:3,4

$\mathrm{A}=-\log \mathrm{T}=\varepsilon \mathrm{bc}$

Teoricamente, qualquer equipamento capaz de detectar variação da intensidade da coloração de uma solução é capaz de possibilitar medidas quantitativas quando a coloração da amostra é comparada com a coloração de soluções de referência com concentrações conhecidas do analito.

Uma substância colorida absorve seletivamente comprimentos de onda da região visível do espectro eletromagnético. A coloração resultante é a cor complementar e é composta pela combinação dos comprimentos de ondas restantes. Durante o processo de absorção, a energia é seletivamente transferida para moléculas e íons e promove elétrons de valência do estado fundamental para estados mais energéticos.

Uma imagem pode ser classificada como um resultado de um estímulo luminoso. Quando uma fotografia ou uma cena real é observada, o olho humano associa os comprimentos de onda refletidos com os padrões de cores e o cérebro humano interpreta as imagens. O digitali- 
zador de uma imagem trabalha de modo similar: a cena real é capturada por um dispositivo eletrônico, tal como uma máquina fotográfica ou um scanner, e o sinal é convertido em uma forma numérica. Na forma digital, as imagens podem ser quantificadas, manipuladas e transformadas matematicamente usando-se programas computacionais.

O caminho mais usado para conversão de uma cena real para uma forma numérica consiste na delimitação de uma área que é dividida em várias partes situadas em um arranjo bidimensional. ${ }^{5} \mathrm{Um}$ ponto de tal arranjo é chamado de pixel.

A representação de cor mais usada foi proposta por um pesquisador da Kodak chamado Bryce Bayer e é conhecida como padrão Bayer. ${ }^{6}$ Essa representação baseia-se no uso de três filtros de cores, vermelho, verde e azul. A combinação das intensidades dessas cores possibilita a geração de diferentes cores.

Para uma imagem digital ser o mais parecida possível com sua imagem original é necessário um número adequado de pixels, que deve ser capaz de representar os detalhes de um gráfico em uma determinada área. A capacidade de representar uma imagem com exatidão é chamada de resolução. Imagens com alta resolução contêm uma grande quantidade de informações.

Em geral, são utilizadas três matrizes, cada uma com um sensor, para armazenar as informações de uma imagem. Os valores da cor vermelha são armazenados na matriz $\mathrm{R}$ (red), da cor verde na matriz $\mathrm{G}$ (green) e da cor azul na matriz B (blue). Esses padrões são chamados de RGB. Como o armazenamento é restrito, a intensidade de radiação é armazenada com 8 bits, totalizando 256 níveis para a região do espectro visível. A combinação das três matrizes ( $R, G$ e B) permite a aquisição de 16 milhões de possibilidades de cores.

A digitalização de uma imagem por um scanner geralmente usa uma barra de radiação que atinge a imagem, transferindo radiação suficiente para o sensor. Com a reflexão da radiação, o sensor pode reconstituir e armazenar a imagem digital.

A resposta analítica que uma imagem gera representa os seus padrões de cores: vermelho, verde e azul (RGB), com valores ${ }^{7}$ entre 0 e 255 . O modelo RGB é um modelo somatório das cores que usa os valores de três cores primárias para fornecer a cor exibida. A variação dessas três cores pode formar outras cores do espectro eletromagnético $(380-730 \mathrm{~nm})$.

Nos últimos anos, o uso de sistemas digitais para aquisição de imagens para o controle de qualidade de produtos vem ganhando espaço na indústria devido ao baixo custo de implementação, possibilidade de monitoramento em linha ${ }^{8} \mathrm{e}$, principalmente, a avaliação de outros parâmetros além das cores, como a distribuição das cores na superfície, a forma e textura dos objetos. ${ }^{9}$

Du e Sun ${ }^{9}$ mostraram em um trabalho de revisão a aplicação de imagens digitais para a definição de padrões de qualidade, como tamanho, forma, cor e textura em alimentos. Entre os vários trabalhos selecionados pelos autores, os alimentos foram classificados em frutos do mar, frutas, grãos, vegetais, pizzas, bolachas e bolos. A forma e as cores (RGB e outras técnicas) dos alimentos foram avaliadas e o tratamento matemático das imagens digitais foi realizado utilizandose ferramentas como análise multivariada e outras técnicas.

Antonelli et al. ${ }^{8}$ descreveram o uso de cores para classificar a qualidade de amostras de "pesto", um típico molho italiano preparado com ervas. Os autores usaram uma máquina fotográfica para a aquisição das imagens digitais. A informação da cor foi analisada por procedimentos quimiométricos. As imagens foram decompostas em um diagrama de cores, que consistia no rearranjo das cores em sequiência para gerar um espectro contínuo. Dessa forma, as imagens puderam ser tratadas da mesma forma que em medidas espectrofotométricas por calibração multivariada. As amostras foram devidamente classificadas e a degradação da clorofila foi detectada com variações de cores imperceptíveis ao olho humano.
Kompany-Zareh et al. ${ }^{10}$ determinaram quantitativamente Fe(II) em amostras de aço usando teste de toque. As imagens foram adquiridas por um scanner de mão e a calibração foi efetuada usando rede neural. Ferro(II) foi quantitativamente recuperado em todas as amostras.

Ferro(III) e Al(III) foram simultaneamente determinados em amostras de aço com recuperação entre 92 e $115 \%$ usando calibração com rede neural. ${ }^{7}$ Cinqüenta recipientes plásticos foram posicionados em um recipiente luminoso com lâmpadas brancas e as soluções foram fotografadas com o uso de uma câmera digital.

Câmera digital também foi utilizada para capturar imagens provenientes de um dermoscópio e de um microscópio em exames dermatológicos para o diagnóstico de doenças dermatológicas. ${ }^{11}$ Em outra aplicação, Behrens e Diepenbrock ${ }^{12}$ utilizaram câmera digital para capturar imagens de canola (Brassica napus) durante o inverno alemão, possibilitando a determinação da estrutura da copa das plantas, bem como sua distribuição sobre o solo e o número de folhas.

Recentemente, Gaião et al..$^{13}$ propuseram o uso de uma WebCam para a determinação de $\mathrm{HCl}$ e $\mathrm{H}_{3} \mathrm{PO}_{4}$ em soluções aquosas e da alcalinidade total em amostras de água mineral e água de torneira. $\mathrm{O}$ sinal monitorado nas titulações foi calculado utilizando-se o sistema de cor RGB para cada imagem adquirida. Os resultados obtidos foram similares aos gerados por titulação espectrofotométrica.

Do ponto de vista didático, um retroprojetor também já foi empregado para medidas espectrofotométricas. ${ }^{14}$ Mais recentemente, demonstrou-se a viabilidade do uso de um scanner para a determinação de mercúrio, obtendo-se um limite de detecção de $7 \mu \mathrm{g} \mathrm{kg}^{-1}$ para a reação colorida envolvendo vapor de mercúrio e iodeto cuproso. ${ }^{15}$ A análise digital da imagem da cor desenvolvida foi usada para estabelecer uma relação quantitativa entre os componentes da cor e a concentração de mercúrio. Os dados foram tratados usando o modelo HLS (Hue-Lightness-Saturation).

O objetivo deste trabalho foi propor um método para determinação de ácido ascórbico em medicamentos utilizando um scanner de mesa para aquisição das imagens. Imagens digitalizadas da amostra foram convertidas numericamente utilizando o padrão de cores RGB. O método proposto pode ser utilizado em aulas de química analítica experimental em combinação com métodos espectrofotométricos bem estabelecidos, ou em substituição a esses quando os equipamentos não estiverem disponíveis.

\section{PARTE EXPERIMENTAL}

\section{Reagentes e preparo das soluções}

Dois medicamentos líquidos (amostras 1 e 2), utilizados em casos de deficiência ou aumento das necessidades de vitamina $C$, foram adquiridos em drogarias locais. Os medicamentos continham uma concentração rotulada de $200 \mathrm{mg} \mathrm{mL}^{-1}$ de ácido ascórbico. O método colorimétrico do azul da Prússia ${ }^{1,2}$ foi utilizado com o intuito de gerar uma solução colorida.

Inicialmente preparou-se $50 \mathrm{~mL}$ de uma solução $10000 \mathrm{mg} \mathrm{L}^{-1}$ de $\mathrm{K}_{3}\left[\mathrm{Fe}(\mathrm{CN})_{6}\right]$ (Merck) em meio de $0,014 \mathrm{~mol} \mathrm{~L}^{-1}$ de $\mathrm{HNO}_{3}$ (Synth) pesando-se 0,502 $\mathrm{g}$ do sal. Em seguida, preparou-se $100 \mathrm{~mL}$ de uma solução $1000 \mathrm{mg} \mathrm{L}^{-1}$ do íon $\mathrm{Fe}(\mathrm{III})$ pesando-se $0,480 \mathrm{~g}$ de $\mathrm{FeCl}_{3} \cdot 6 \mathrm{H}_{2} \mathrm{O}$ (Merck) e completando-se o volume com a adição de água destilada e desionizada em sistema Milli- ${ }^{\circledR}$ (Millipore). Preparou-se também $50 \mathrm{~mL}$ de uma solução $1761,7 \mathrm{mg} \mathrm{L}^{-1}$ de ácido ascórbico $\left(\mathrm{C}_{6} \mathrm{H}_{8} \mathrm{O}_{6}\right)$ (Mallinckrodt) em meio de $0,014 \mathrm{~mol} \mathrm{~L}^{-1}$ de $\mathrm{HNO}_{3}$ pesando-se 88,08 mg do composto.

Duas amostras de vitamina C comerciais, de diferentes laboratórios, foram utilizadas. Baseando-se nos valores de ácido ascórbico rotulados, soluções com concentrações aproximadas de 1700 mg 
$\mathrm{L}^{-1}$ das amostras 1 e 2 foram preparadas em meio $0,014 \mathrm{~mol} \mathrm{~L}^{-1}$ de $\mathrm{HNO}_{3}$ adicionando-se $85 \mu \mathrm{L}$ das amostras em um recipiente no qual o volume final da solução foi de $10 \mathrm{~mL}$. Como branco analítico foi utilizada solução $0,014 \mathrm{~mol} \mathrm{~L}^{-1}$ de $\mathrm{HNO}_{3}$.

\section{Instrumentação}

Um scanner de mesa Hewlett-Packard modelo Scanjet 2400, 1200 dpi foi empregado na digitalização das análises. As imagens digitais obtidas foram tratadas e quantificadas matematicamente utilizando como programa computacional Adobe Photoshop versão 7.0.

Para a comparação dos resultados, as amostras também foram analisadas usando um espectrofotômetro na região do visível da marca FEMTO, modelo 432.

\section{Procedimento}

Tanto o método empregando imagem digital quanto o método espectrofotométrico se basearam na reação entre hexacianoferrato de potássio, cloreto de ferro (III) e ácido ascórbico, respectivamente, na proporção 1:2:2 $\mathrm{V} \mathrm{v}^{-1}$ para a formação do complexo do azul da Prússia. Comprimentos de onda na região de $700 \mathrm{~nm}$ foram empregados para medidas de absorbância no procedimento espectrofotométrico.

Para a aquisição das imagens digitais, as soluções foram adicionadas em um recipiente plástico adquirido no comércio local e usualmente empregado para a fabricação de bombons de chocolate (Figura 1). Cada recipiente plástico continha 11 pequenos orifícios cilíndricos com volume de aproximadamente $8 \mathrm{~mL}$.

A aquisição das imagens foi realizada em três etapas, a primeira delas para a curva analítica e as outras para as amostras dos medicamentos com volume normal $(7,0 \mathrm{~mL})$ e com volume reduzido $(3,5$ $\mathrm{mL})$. O volume de amostra adicionado sobre cada cavidade da fôrma

a)

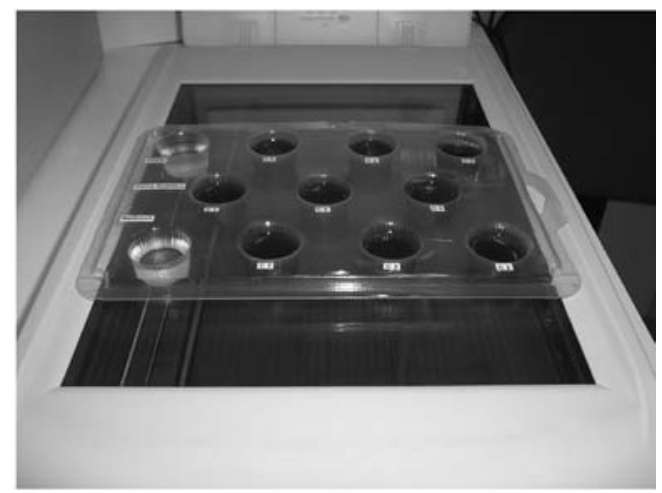

b)

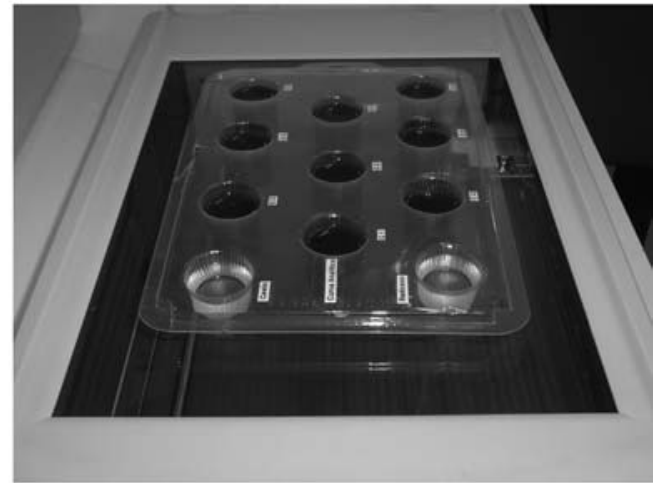

Figura 1. Posicionamento da fôrma sobre o scanner. a) Paralelamente ao feixe de luz e b) perpendicularmente ao feixe de luz foi de 7,0 e 3,5 mL (altura de aproximadamente $1 \mathrm{~cm}$ para o maior volume e $0,5 \mathrm{~cm}$ para o menor volume). Esse experimento equivale à variação do caminho óptico do meio absorvente. Após o posicionamento da fôrma sobre a superfície do scanner devidamente nivelado horizontalmente, esse recipiente foi coberto com um papel branco e a amostra foi digitalizada. Após a digitalização das amostras, uma área de $100 \times 100$ pixels da imagem obtida foi selecionada utilizando-se o programa de tratamento de imagens Adobe Photoshop. Os valores médios das cores primárias, vermelha (R), verde $(\mathrm{G})$ e azul (B) foram calculadas com esse mesmo programa para cada amostra selecionada. Os valores RGB obtidos para as amostras foram comparados com valores obtidos para as soluções de referência.

A curva analítica para análise por imagem digital foi preparada utilizando-se 50; 100 e $150 \mathrm{mg} \mathrm{L}^{-1}$ de ácido ascórbico (c1, c2 e c3, respectivamente).

\section{Conversão dos valores de RGB em absorbância}

$\mathrm{Na}$ espectrofotometria de absorção molecular um feixe de radiação monocromático com potência " $\mathrm{P}_{0}$ " atravessa uma solução e parte desse é absorvido, sofrendo uma atenuação, denominada como "P". A relação entre $\mathrm{P}$ e $\mathrm{P}_{0}$ fornece os valores de transmitância, ou porcentagem de radiação absorvida.

As soluções digitalizadas por um scanner de mesa comum realizam processo análogo ao espectrofotômetro, em que um feixe de radiação $\mathrm{P}_{0}$ (barra de radiação) incide no recipiente de amostra sofrendo uma absorção parcial e retorna para o sensor que determina qual a cor resultante (P). No padrão de cor RGB, a cor branca equivale ao valor máximo de 255 .

A partir das cores de um branco analítico $\left(\mathrm{P}_{0}\right)$ e das cores das amostras é possível calcular o valor de transmitância das soluções e, conseqüentemente, o valor de absorbância. Para a determinação do teor de ácido ascórbico foi avaliada a cor Azul (B). O complexo azul da Prússia faz com que a cor azul do padrão RGB sofra crescente atenuação com o aumento da concentração. Neste trabalho, não foi utilizada a cor vermelha porque essa cor se encontrava saturada nas imagens obtidas.

\section{Posição da fôrma sobre o scanner}

Com o intuito de avaliar a melhor posição dos frascos no scanner e a repetibilidade das medidas, o recipiente (fôrma) foi posicionado perpendicularmente ou paralelamente à barra de luz do scanner e as soluções foram digitalizadas. A Figura 1 apresenta ambos posicionamentos da fôrma utilizados. Nas duas situações as soluções c1, c2, c3 apresentavam as mesmas concentrações para as posições perpendicular ou paralela. É importante ressaltar que em ambos experimentos manteve-se o nivelamento horizontal do scanner.

\section{RESULTADOS E DISCUSSÃO}

\section{Avaliação do efeito da posição da fôrma sobre o scanner e do volume de solução}

Inicialmente foi avaliado o melhor posicionamento dos frascos na superfície do scanner. Com o intuito de gerar imagens com pequeno tamanho (aproximadamente $100 \mathrm{~kb}$ ) a resolução utilizada nas análises foi de 200 dpi em todos os experimentos realizados.

As soluções foram adicionadas na fôrma e essa foi colocada sobre a superfície do scanner, de modo que ficasse posicionada perpendicular ou paralelamente ao feixe de luz do scanner. Foram analisadas 11 soluções simultaneamente, sendo que 3 continham as soluções de referência; três contendo a amostra 1 e outras três contendo a amostra 
2, e as últimas 2 contendo o branco analítico. A Tabela 1 mostra as concentrações de ácido ascórbico obtidas posicionando-se a fôrma paralelamente ou perpendicular sobre o scanner com volumes normais e reduzidos das soluções.

Tabela 1. Teores de ácido ascórbico $\left(\mathrm{mg} \mathrm{L}^{-1}\right)$ determinados para diferentes posicionamentos da fôrma, perpendicular ou paralelamente, sobre a superfície do scanner com volumes normal e reduzido de amostra. Resolução de 200 dpi

\begin{tabular}{lcc}
\hline $\begin{array}{l}\text { Posicionamento da fôrma sobre o } \\
\text { scanner }\end{array}$ & $\begin{array}{c}\mathrm{B}^{*} \\
\text { (Amostra 1) }\end{array}$ & $\begin{array}{c}\text { B* } \\
\text { (Amostra 2) }\end{array}$ \\
\hline $\begin{array}{l}\text { Paralelamente ao feixe de luz/ } \\
\text { volume normal }\end{array}$ & $202,3 \pm 2,3$ & $220,6 \pm 2,3$ \\
$\begin{array}{l}\text { Paralelamente ao feixe de luz/ } \\
\text { volume reduzido }\end{array}$ & $212,6 \pm 5,3$ & $236,0 \pm 4,1$ \\
$\begin{array}{l}\text { Perpendicularmente ao feixe de luz/ } \\
\text { volume normal }\end{array}$ & $219,6 \pm 1,4$ & $226,9 \pm 1,8$ \\
$\begin{array}{l}\text { Perpendicularmente ao feixe de luz/ } \\
\text { volume reduzido }\end{array}$ & $211,4 \pm 4,9$ & $241,2 \pm 2,1$ \\
\begin{tabular}{l} 
Espectrofotômetro \\
\hline
\end{tabular} & $242,3 \pm 3,5$ & $253,5 \pm 3,5$ \\
\hline
\end{tabular}

* média \pm desvio padrão $(\mathrm{n}=5)$; $\mathrm{B}($ blue $)$ : corresponde à cor azul gerada pela formação do complexo azul da Prússia

Os desvios padrão obtidos foram menores para as amostras posicionadas perpendicularmente para ambos os medicamentos. Além disso, amostras com volumes menores de solução tiveram desvios padrão maiores que os correspondentes aos volumes maiores.

Quando a fôrma foi posicionada paralelamente, foi possível observar que parte da luz do scanner foi refletida nas partes mais externas da fôrma. Essa reflexão afetou os cálculos matemáticos e causou erros nas quantificações. Entretanto, o posicionamento da fôrma perpendicularmente ao scanner evitou a reflexão da luz e os desvios padrão obtidos foram menores que os valores correspondentes ao posicionamento paralelo, tanto nos volumes maiores quanto nos volumes reduzidos para ambas amostras.

A seleção da área da imagem para cálculo da cor é mais difícil quando ocorre reflexão. Entretanto, o posicionamento da fôrma perpendicularmente e centralizado no scanner evitou a reflexão e os desvios padrão obtidos foram menores que $5 \%$.

\section{Lei de Beer}

Para avaliar a influência do volume de amostra nas medidas analíticas e verificar a aplicação da lei de Beer no sistema proposto duas observações foram realizadas, a primeira procurou avaliar o comportamento do sistema empregando dois volumes de amostra 3,5 e 7,0 mL correspondente a um caminho óptico de 0,5 e 1,0 cm, respectivamente, e o segundo teste avaliou a variação da absorbância com o aumento da concentração de 50 a $100 \mathrm{mg} \mathrm{L}^{-1}$ do complexo azul da Prússia.

Segundo a lei de Beer, quanto maior o caminho óptico que o feixe de radiação tem que percorrer, a uma concentração constante, maior será sua absorção. A Figura 2 ilustra a aplicação desta Lei, em que volumes maiores de solução proporcionaram um aumento linear da absorbância, influenciando a sensibilidade da determinação. Efeito similar foi obtido quando o parâmetro caminho óptico foi mantido fixo e a concentração das soluções foi alterada.

A linearidade das medidas e o valor de absortividade molar similar de ambos ensaios demonstram que a Lei de Beer pode ser aplicada para os procedimentos de quantificação utilizando imagens digitais,

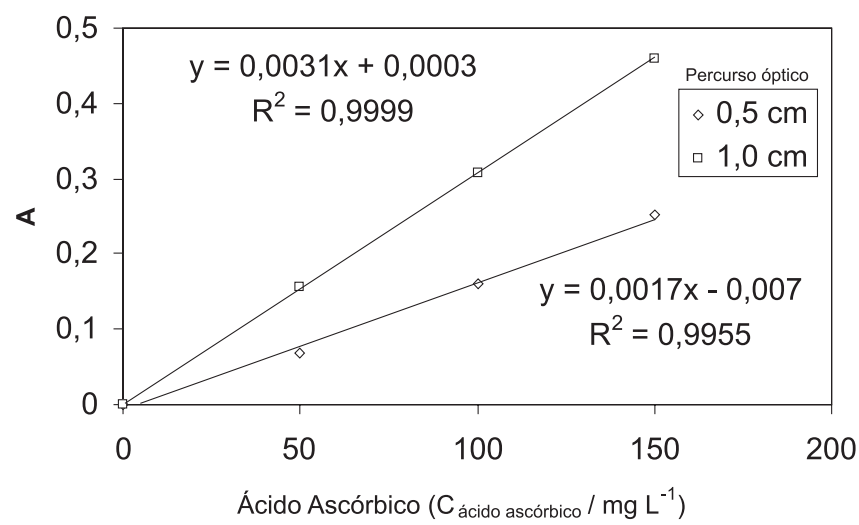

Figura 2. Variação do caminho óptico e da concentração da solução

mesmo usando vários comprimentos de onda para compor a cor das soluções e não apenas um como a Lei de Beer propõe. Isso é possível porque o único composto em solução que absorvia uma fração da radiação visível era o azul da Prússia.

\section{Determinação de ácido ascórbico}

Inicialmente foram preparadas três soluções com concentrações de 50,101 e $151 \mathrm{mg} \mathrm{L}^{-1}$ de ácido ascórbico. A determinação da concentração de ácido ascórbico foi obtida pela interpolação da reta. As diluições dos medicamentos foram feitas de modo que as concentrações dessas soluções ficassem próximas da concentração $100 \mathrm{mg} \mathrm{L}^{-1}$.

Empregando-se o programa Adobe Photoshop, os valores para o padrão azul (B) foram determinados para as medidas perpendicularmente ao feixe de luz com volume maior de amostra.

O coeficiente de correlação linear da curva analítica utilizando o scanner de mesa comum foi de 0,9997 com equação linear igual a $\mathrm{Y}=0,00305 \mathrm{X}+0,00272$.

A concentração de ácido ascórbico determinada pela procedimento proposto foi próxima ao valor indicado no rótulo do produto. Uma observação importante é que concentrações de ácido ascórbico em ambos os medicamentos (Tabela 1) foram sempre maiores que os valores encontrados nas bulas dos medicamentos $\left(200 \mathrm{mg} \mathrm{mL}^{-1}\right)$. Esse resultado pode estar relacionado com a possibilidade de ocorrer oxidação do medicamento em função do tempo de armazenagem e a necessidade de garantia do teor rotulado durante o tempo de validade do produto.

A determinação do teor de ácido ascórbico por espectrofotometria de absorção molecular apresentou resultados ligeiramente superiores aos obtidos pelo scanner (Tabela 1). A curva analítica obtida utilizando o espectrofotômetro não foi incluída na Figura 2 devido à diferença da magnitude dos sinais de absorbância. Deve-se ressaltar que o coeficiente angular obtido para o espectrofotômetro foi 10 vezes maior que aquele obtido para as medidas com scanner (caminho óptico de $1 \mathrm{~cm}$ em ambos os casos), o que reflete o comportamento esperado considerando-se a monocromaticidade do feixe de radiação em cada caso.

As vantagens do uso de um scanner nas análises das reações colorimétricas são o baixo custo, a possibilidade de adotar o procedimento para cursos de graduação que não disponham de espectrofotômetro e a consolidação dos conceitos da Lei de Beer.

\section{CONCLUSÃO}

O uso de imagens digitais em análises químicas é uma alternativa para a determinação colorimétrica. A quantificação matemática para as soluções com o complexo do azul da Prússia foi adotada para a 
determinação de vitamina $\mathrm{C}$ nas amostras de dois medicamentos comerciais. O nivelamento e o posicionamento da fôrma sobre a superfície do scanner foi um fator importante. Quando a fôrma foi posicionada perpendicularmente ao feixe de luz foram obtidos melhores resultados devido à menor reflexão da radiação. $\mathrm{O}$ efeito do volume da solução sobre a fôrma também foi importante, sendo que volumes maiores de amostra, i.e., maior caminho óptico, geraram menores desvios padrão. A comparação com o método espectrofotométrico indicou que a análise de imagens digitais pode ser aplicada para a determinação analítica, além disso, o uso de imagens digitais possibilitou análise simultânea de 11 soluções.

Esse experimento pode ser adotado em cursos de análise instrumental e possibilita uma discussão mais ampla sobre espectrofotometria de absorção molecular, considerando-se a fonte de radiação e o tratamento de dados.

\section{AGRADECIMENTOS}

Os autores agradecem ao apoio do Conselho Nacional de Desenvolvimento Científico e Tecnológico (CNPq), à Coordenação de Aperfeiçoamento de Pessoal de Nível Superior (CAPES), à Fundação de Amparo à Pesquisa do Estado de São Paulo (FAPESP, Processos 05/00878-0 e 05/54230-0) e ao Dr. H. J. Vieira pela colaboração nas análises utilizando espectrofotômetro.

\section{REFERÊNCIAS}

1. Vogel, A.; Química Analítica Qualitativa, Editora Mestre Jou: São Paulo, 1981.

2. Nóbrega, J. A.; Lopes, G. S.; Talanta 1996, 43, 971.

3. Harris, D. C.; Quantitative Chemical Analysis, $6^{\text {th }}$ ed., W. H. Freeman: New York, 2003.

4. Skoog, D. A.; Holler, F. J.; Nieman, T. A.; Principles of Instrumental Analysis, $5^{\text {th }}$ ed., Saunders College: Philadelphia, 1998.

5. VanEspen, P.; Janssens, G.; Vanhoolst, W.; Geladi, P.; Analusis 1992, 20, 81.

6. http://www.kodak.com/global/plugins/acrobat/en/digital/ccd/ applicationNotes/Color CorrectionforImageSensors.pdf , acessada em Maio 2006.

7. Maleki, N.; Safavi, A.; Sedaghatpour, F.; Talanta 2004, 64, 830.

8. Antonelli, A.; Cocchi, M.; Fava, P.; Foca, G.; Franchini, G. C.; Manzini, D.; Ulrici, A.; Anal. Chim. Acta 2004, 515, 3.

9. Du, C. J.; Sun, D. W.; Trends Food Sci Technol. 2004, 15, 230.

10. Kompany-Zareh, M.; Mansourian, M.; Ravaee, F.; Anal. Chim. Acta 2002, 471, 97.

11. Rushing, M. E.; Hurst, E.; Sheehan, D.; J. Amer. Acad. Dermat. 2006, 55,314 .

12. Behrens, T.; Diepenbrock, E.; J. Agron. Crop Sci. 2006, 192, 295.

13. Gaião, E. N.; Martins, V. L.; Lyra, W. S.; Almeida, L. F.; Silva, E. C.; Araújo, M. C. U.; Anal. Chim. Acta 2006, 570, 283.

14. Gutz, I. G. R.; Angnes, L.; Pedrotti, J. J.; J. Chem. Educ. 1991, 68, 325.

15. Paciornik, S.; Yallouz, A. V.; Campos, R. C.; Gannerman, D.; J. Braz. Chem. Soc. 2006, 17, 156. 